\title{
RESEARCH
}

\section{Ovarian cancer risk in relation to medical visits, pelvic examinations and type of health care provider}

\author{
Haim A. Abenhaim, Linda Titus-Ernstoff, Daniel W. Cramer
}

$\infty$

See related article page 949

\section{ABSTRACT}

Background: Whether the current recommendations for ovarian cancer prevention and screening (annual history and physical examination) are effective has not been evaluated. We examined the relation between health care use and the risk of ovarian cancer.

Methods: Using a case-control study design, we recorded the frequency of medical visits and pelvic examinations and the type of health care provider visited during a 5-year period from interviews with women with and without ovarian cancer between between July 1998 and July 2003. We used multivariable logistic regression analysis to calculate the adjusted odds ratio of ovarian cancer associated with the frequency of medical visits and pelvic examinations and the type of health care provider. In addition, we stratified cases and controls by menopausal status and cancer histologic subtype and grade.

Results: A total of 668 cases and 721 age-matched controls agreed to participate in the study. We observed an increased risk of ovarian cancer among women who, during the 5-year study period, did not have a medical visit (odds ratio [OR] $2.8,95 \%$ confidence interval $[\mathrm{Cl}] \mathbf{1 . 5}-5.0$ ) or pelvic examination (OR 3.9, 95\% Cl 2.2-6.9) or who had no regular health care provider (OR 2.7, 95\% Cl 1.3-5.7). This increase in risk was most pronounced among women who were postmenopausal (no medical visit, OR 7.7, 95\% Cl 2.6-23.0; no pelvic examination, $\mathrm{OR} 3 \cdot 3,95 \% \mathrm{Cl} 1.7-6.0$; no health care provider, OR $12.5,95 \% \mathrm{Cl} 2.7-57 \cdot 5$ ).

Interpretation: Although the exact mechanism underlying the association between medical visits, pelvic examinations and type of health care provider and ovarian cancer is unknown, women should be encouraged to maintain regular medical care.

CMAJ 2007;176(7):94I-7

\} $\mathrm{n}$ North America, ovarian cancer is the second most common gynecologic malignant disease and is the leading cause of death among women with gynecologic cancer. ${ }^{1}$ The high case-fatality rate is in part due to delayed diagnosis and to poor survival of women who have advanced disease. Several studies have examined the efficacy of routine ultrasonography and screening for cancer antigen I25 for early detection of ovarian cancer; however, these techniques have not been clearly shown to be effective for early detection..$^{2-8}$ As a consequence, the American College of Obstetricians and Gynecologists does not recommend the routine use of these techniques for ovarian cancer screening, but it does recommend that women have an annual history and pelvic examination. ${ }^{9}$ However, the value of even these measures as a part of preventive health care has not been well established. In our study, we assessed the frequency of medical visits and pelvic examinations and the type of health care provider visited among women with and without ovarian cancer in order to assess the risk of ovarian cancer in relation to these aspects of health care.

\section{Methods}

We performed a case-control study of ovarian cancer from July I998 to July 2003 in eastern Massachusetts and all of New Hampshire. This study was approved by the Institutional Review Boards of the Brigham and Women's Hospital and the Dartmouth Medical Center. We identified I267 cases from tumour boards and statewide registries, both of which are established methods to identify cancer cases. ${ }^{10,11}$ We excluded II9 patients who had died, IIo who had moved away from the study area, I who had no telephone, 23 who did not speak English and 46 who, upon review, were found to have a non-ovarian primary tumour. Of the remaining 968 cases, permission to contact 106 patients was denied by their physicians and I7I patients declined to participate, which left 69I patients available for interview. Of these patients, $668 \mathrm{had}$ epithelial ovarian cancer (including borderline malignant disease) and were included in our study.

Control patients were identified through town books in Massachusetts and driver licence lists in New Hampshire. We performed frequency matching by selecting controls from these lists to match the age and area of residence of cancer cases. The validity of this method for selecting controls has been shown to be an effective means of population sampling. ${ }^{12,13}$ We sent invitations to I 843 women to participate in the study as controls. Of these women, 3 I 8 had moved, could not be located or had died, I97 (in Massachu- 
setts) returned an "opt out" postcard (sent to prospective controls as a requirement of the Institutional Review Boards) and 47 no longer had a working telephone. Of the remaining I28I women, I52 were ineligible because they had previously undergone an oophorectomy or were not the correct age, 59 were incapacitated or did not speak English and 349 declined to participate, which left 72I women who were interviewed and included in our study.

After we obtained written informed consent, an in-person interview was conducted to ask participants questions about their demographic characteristics and their medical and family history. Participants also completed a self-administered dietary questionnaire. They were unaware of specific hypotheses; we assessed exposure before a reference date (I year before the date of diagnosis for cases and the date of the interview for controls). Participants were questioned about the frequency of medical visits and pelvic examinations in the 5year period before the reference date and about the type of health care provider that they visited most regularly. Cases and controls were categorized by the frequency of medical visits ( $\geq 5,3-4, \mathrm{I}-2$ or no visit); by the frequency of pelvic examinations ( $\geq 5,3-4, \mathrm{I}-2$ or no examination); and by the type of health care provider most regularly visited (obstetriciangynecologist, other physician [internist or family physician], non-physician [e.g., chiropractor, nurse-practitioner, homeopathic or holistic practitioner, physician's assistant] or no health care provider).

To describe factors associated with health care use among controls, we compared the baseline characteristics of control subjects who had at least I medical visit and at least I pelvic examination per year and who had an obstetriciangynecologist as their regular care provider with the characteristics of controls who did not have this type of health care. We evaluated 2 types of baseline characteristics: those that are not affected by health care encounters (age, ethnic background, religion, education, marital status, parity and family history of breast or ovarian cancer) and those that may be or are likely to be affected by the health care encounter (smoking, medication use, multivitamin use, use of talc in the genital area and gynecologic surgery).

We used unconditional multivariable logistic regression models to estimate the risk of ovarian cancer by frequency of medical visits and pelvic examinations and by type of health care provider. An initial model that included known confounders was used to evaluate the relation between the frequency of health care visits and risk of ovarian cancer. A second model that included additional potential causal intermediates was used to evaluate the role of these factors in relation to frequency of health care visits and ovarian cancer. All statistical tests were 2 -tailed, and a $p$ value of $\leq 0.05$ was considered statistically significant.

We categorized medications as either prescription (antihypertensives, antidepressants, antihistamines and thyroid medications) or over-the-counter (mainly analgesics). We conducted subgroup analyses according to menopausal status and histologic subtype of ovarian cancer. Women were considered postmenopausal if they reported an absence of menses for at least I 2 months. For women who had a hys- terectomy before menstruation ceased, menopausal status was determined by age (premenopausal if less than 50 years of age, postmenopausal if 50 years or older). Interaction terms were used in the analyses to evaluate effect modification by menopausal status.

\section{Results}

In this age-matched cohort, cases were more likely than controls to be nulliparous, to be unmarried and to have a family history of breast or ovarian cancer. However, cases were less likely than controls to have ever taken oral contraceptive pills or hormone replacement therapy or to have had a tubal ligation. Detailed descriptions of the baseline characteristics of the cases and controls have previously been published. ${ }^{14}$ Table I and Table 2 describe key demographic and medical characteristics of the participants, stratified by whether or not they had at least I medical visit per year, at least I pelvic examination per year or if their regular health care provider was an obstetrician-gynecologist. Cases were less likely than controls to report at least I medical visit per year ( $7 \mathrm{I} .0 \% \mathrm{v} .76 .3 \%)$ or at least I pelvic examination per year $(67.7 \%$ v. $74.5 \%)$. There was no difference between the proportion of cases and controls who chose an obstetrician-gynecologist as their regular care provider. For the majority of characteristics, cases were less likely than controls to routinely use health care services. Compared with cases who had at least I pelvic examination per year, controls with this frequency of pelvic examinations were younger and were more likely to have more than a high school education, to have a body mass index of less than $30 \mathrm{~kg} / \mathrm{m}^{2}$, to have taken prescription medications, oral contraceptive pills and hormone replacement therapy, to have had a tubal ligation or any gynecologic surgery and to have a family history of breast cancer. Among participants whose regular care provider was an obstetrician-gynecologist, controls were more likely than cases to be younger, to be married, to have taken prescription medications, to have a history of hormone replacement therapy and to have had an ovarian cystectomy, but they were less likely to use talc for genital hygiene.

Table 3 presents the adjusted odds ratios of ovarian cancer associated with the frequency of medical visits and pelvic examinations and the type of health care provider. Compared with controls, a larger proportion of cases had no medical visit in the 5 -year period ( $6.1 \%$ v. $2.4 \%$ ), no pelvic examination ( $9.4 \%$ v. $2.5 \%)$ and no regular health care provider ( $4.9 \%$ V. I. $5 \%)$. The risk of ovarian cancer was increased among women who had no medical visit (adjusted odds ratio [OR] 2.8, 95\% confidence interval [CI] I.5-5.0) or pelvic examination (OR 3.9, 95\% CI 2.2-6.9) or had no regular health care provider (OR 2.7, 95\% CI I.3-5.7). There was no increased risk of ovarian cancer among women who had I-2 or 3-4 medical visits or pelvic examinations during the 5-year period. The risk of ovarian cancer was highest among postmenopausal women (no medical visits, OR 7.7, 95\% CI 2.6-23.0; no pelvic examination, OR 3.3, 95\% CI I.7-6.5 ; no regular care provider, OR I2.5, 95\% CI2.7-57.6). After we 
adjusted for potential intermediates in the causal pathway (smoking, body mass index, hormone replacement therapy, oral contraceptive pills, over-the-counter medications, prescription medications, multivitamins, talc for genital hygiene, tubal ligation and ovarian cystectomy), we found a marked attenuation of the effect of health care on the risk of ovarian cancer (likelihood ratio test: no medical visits $p<$ o.ooI; no pelvic examinations $p<0.001$; no regular care provider $p<0.00 \mathrm{I})$. The proportion of women who had no pelvic examination in the 5-year study period differed by type of regular care provider: obstetrician-gynecologist $\mathrm{I} \%$, other physician $4 \%$, non-physician $14 \%$ and no regular care provider $66 \%$.

Among the cases, the most common histologic subtype was serous invasive ovarian cancer $(26 \mathrm{I}[39 . \mathrm{I} \%])$; the next most common were (20I [30.I\%]), serous borderline (9I $[13.6 \%])$ and mucinous (73 [10.9\%]). In general, the increased risk of ovarian cancer associated with no medical

Table 1: Demographic characteristics of women with ovarian cancer $(n=668)$ and age-matched controls $(n=721)$

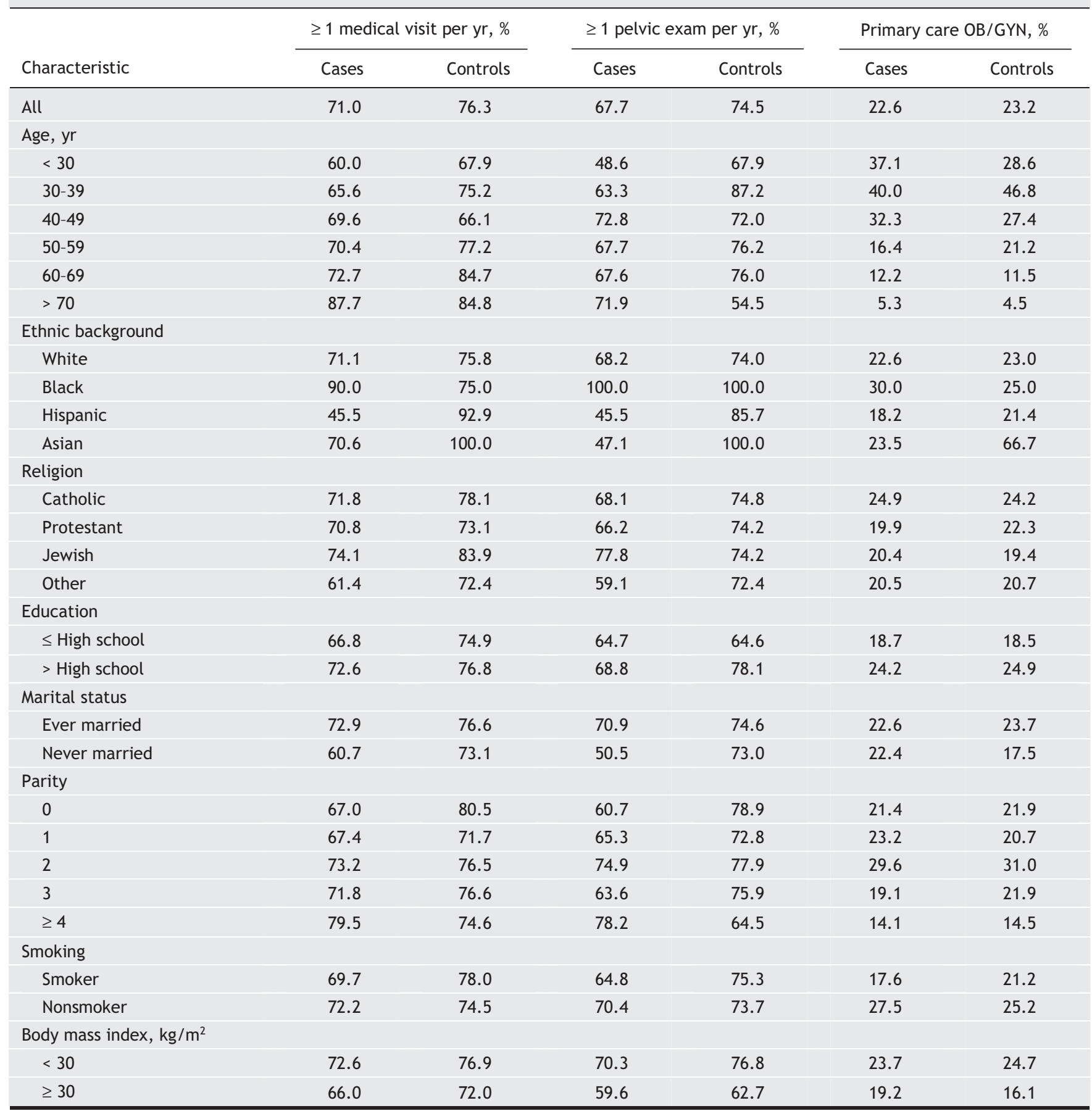

Note: $\mathrm{OB} / \mathrm{GYN}=$ obstetrician-gynecologist. 
Table 2: Medical history of cases $(n=668)$ and controls $(n=721)$

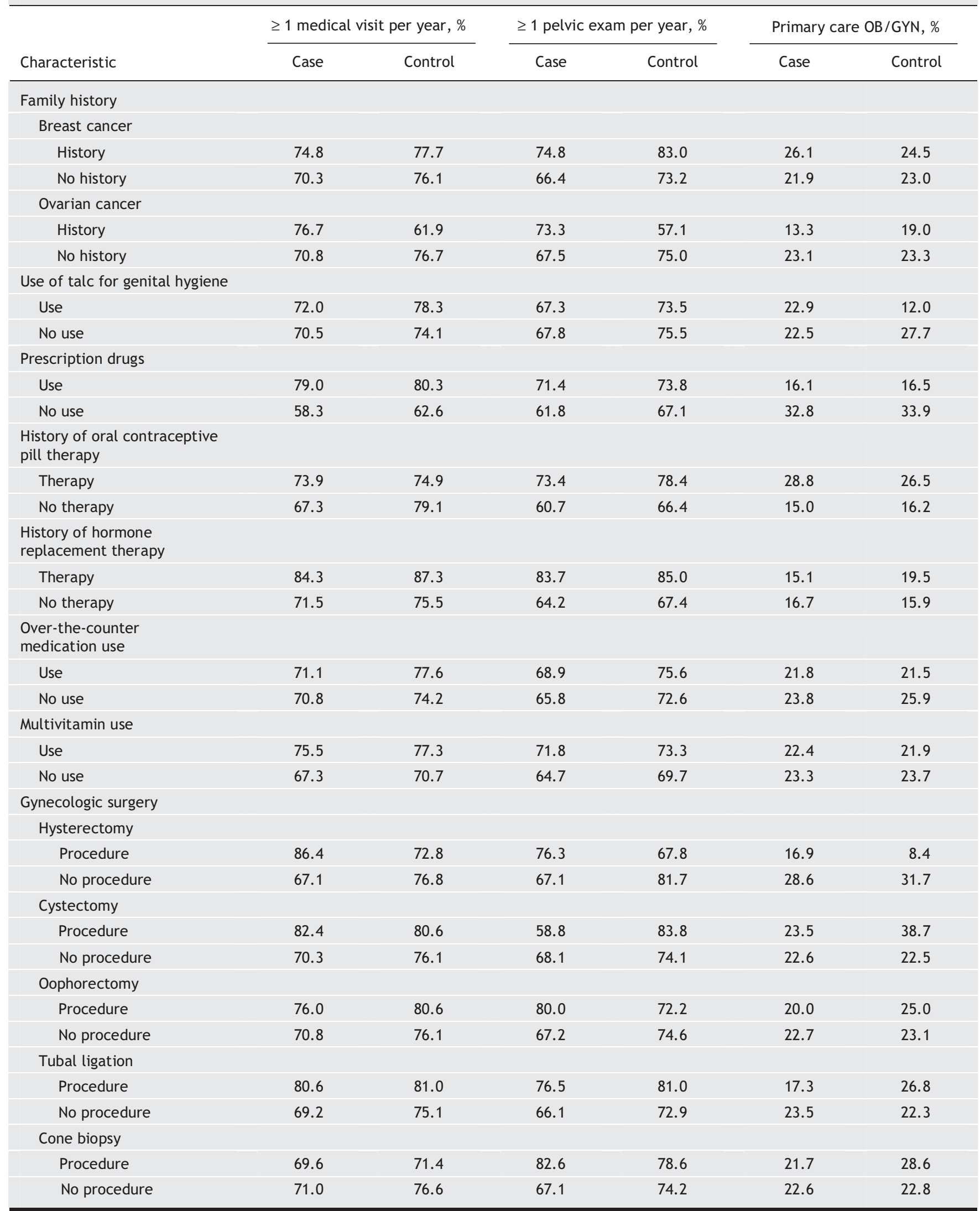

Note: $\mathrm{OB} / \mathrm{GYN}=$ obstetrician-gynecologist. 
visits or pelvic examinations was consistent for different histologic subtypes and grades of ovarian cancer. There was no obvious change in effect when cases were stratified by menopausal status (data not shown).

\section{Interpretation}

In our study, we found that women who had no medical visits, pelvic examinations or regular health care provider were at increased risk of ovarian cancer. The risk was most apparent among postmenopausal women, especially those with no regular health care provider.

When interpreting these findings, it is first necessary to consider the possibility of bias or confounding, especially in the context of a case-control study. Because not all of the potential controls who were contacted agreed to participate in the study (about a $70 \%$ response rate), we could not exclude the possibility that the controls who chose to participate were more likely than those who did not participate to be concerned about their health and were thus more likely to be regular health care users. The key issue then was to determine whether the pattern of medical visits and pelvic examinations among the controls in our study was representative of the pattern in the general population or whether this introduced an element of selection bias. In a 200 I US study involving 3966 women aged I8-64, 75\% reported having I or more pelvic examinations per year and $3 \%$ reported "not usually getting these exams." ${ }^{15}$ In our study, $74 \%$ of the control subjects had annual or more frequent pelvic examinations and $2 \%$ had no pelvic examinations during the 5 -year study period, which suggests that the pattern of health care use in our control group was a reasonable reflection of the pattern of use in the general population.

Selection bias is also a concern in the case group. Since we were unable to include all patients with ovarian cancer in New England, it is possible that the health care practices of the participants were not representative of the practices of the total population of patients with ovarian cancer. Among the pool of eligible patients, a considerable proportion was excluded because their physician declined permission to contact the patient, which may have possibly introduced selection bias. To evaluate this, we stratified our results by location. When the results from New England (where physicians were more likely to decline permission

Table 3: Association between the risk of ovarian cancer and frequency of medical visits and pelvic examinations and type of regular health care provider, stratified by menopausal status

\begin{tabular}{|c|c|c|c|c|c|c|c|c|c|}
\hline Variable & \multicolumn{3}{|c|}{ All participants } & \multicolumn{3}{|c|}{ Premenopausal women } & \multicolumn{3}{|c|}{ Postmenopausal women } \\
\hline \multicolumn{10}{|l|}{$\begin{array}{l}\text { No. of medical } \\
\text { visits }\end{array}$} \\
\hline $1-2$ & 96 & 100 & $1.1(0.8-1.6)$ & 55 & 59 & $1.1(0.7-1.7)$ & 41 & 41 & $1.2(0.7-1.9)$ \\
\hline 0 & 41 & 17 & $2.8(1.5-5.0)$ & 15 & 13 & $1.2(0.5-2.7)$ & 26 & 4 & $7.7(2.6-23.0)$ \\
\hline $0^{\dagger}$ & 41 & 17 & $2.4(1.3-4.7)$ & 15 & 13 & $1.1(0.4-2.8)$ & 26 & 4 & $5.6(1.8-17.3)$ \\
\hline \multicolumn{10}{|l|}{$\begin{array}{l}\text { No. of pelvic } \\
\text { examinations }\end{array}$} \\
\hline 0 & 63 & 18 & $3.9(2.2-6.9)$ & 23 & 5 & $5.0(1.8-14.2)$ & 40 & 13 & $3.3(1.7-6.5)$ \\
\hline $0^{\dagger}$ & 63 & 18 & $2.9(1.6-5.3)$ & 23 & 5 & $3.3(1.1-9.9)$ & 40 & 13 & $2.3(1.1-4.7)$ \\
\hline \multicolumn{10}{|l|}{$\begin{array}{l}\text { Health care } \\
\text { provider }\end{array}$} \\
\hline OB/GYN & 151 & 167 & 1.0 & 104 & 112 & 1.0 & 47 & 55 & 1.0 \\
\hline $\begin{array}{l}\text { Other } \\
\text { physician }\end{array}$ & 463 & 519 & $0.9(0.7-1.2)$ & 181 & 199 & $0.8(0.5-1.1)$ & 282 & 320 & $1.1(0.7-1.7)$ \\
\hline Non-physician & 19 & 24 & $0.8(0.4-1.6)$ & 12 & 17 & $0.6(0.3-1.3)$ & 7 & 7 & $1.4(0.4-4.3)$ \\
\hline No provider & 33 & 11 & $2.7(1.3-5.7)$ & 12 & 9 & $0.8(0.3-2.1)$ & 21 & 2 & $12.5(2.7-57.6)$ \\
\hline No provider ${ }^{\dagger}$ & 33 & 11 & $2.2(1.0-4.8)$ & 12 & 9 & $0.8(0.3-2.4)$ & 21 & 2 & $7.4(1.5-36.1)$ \\
\hline
\end{tabular}

Note: $\mathrm{OR}=$ odds ratio, $\mathrm{Cl}=$ confidence interval, $\mathrm{OB} / \mathrm{GYN}=$ obstetrician-gynecologist.

*Adjusted for age, parity, ethnic background, education, marital status, religion and family history of breast or ovarian cancer.

†Adjusted additionally for smoking, body mass index, hormone replacement therapy, oral contraceptive pills, over-the-counter medications, prescription medications, multivitamins, talc for genital hygiene, tubal ligation and ovarian cystectomy. 
for their patients to be contacted) were compared with those from New Hampshire (where almost all physicians agreed to allow their patients to be contacted), we obtained similar point estimates, which suggests that the selective loss of case subjects by physician refusal was not likely a source of bias.

We next considered the important issue of confounding. First, we evaluated variables that would not be changed by, but might influence, health care use. These potential confounders were age, ethnicity, marital status, parity, religion and family history and were included as adjustment variables in all logistic models. Second, we evaluated variables that, unlike true confounders, may be elements in the causal pathway between health care use and ovarian cancer. Variables in this category may be influenced by health care encounter and included body mass index, use of talc for genital hygiene, use of over-the-counter medications, use of prescription drugs and pelvic surgery. We examined the potential for these variables to influence the association between health care use and ovarian cancer risk by determining whether these variables were associated with health care use. Not surprisingly, prescription drug use, including hormonal replacement therapy and oral contraceptives, use of talc for genital hygiene and history of tubal ligation and ovarian cystectomy were associated with health care use and type of regular health care provider. Importantly, some of these variables (smoking, ${ }^{16}$ use of talc for genital hygiene, ${ }^{17,18}$ body mass index ${ }^{19}$ and hormone replacement therapy ${ }^{20,21)}$ have previously been found to be associated with increased ovarian cancer risk. However, other variables such as tubal ligation, ${ }^{22,23}$ use of oral contraceptives, ${ }^{24,25}$ use of over-the-counter medications, ${ }^{14}$ use of vitamin supplements ${ }^{26,27}$ and certain types of prescription medications (e.g., cholesterol-lowering agents) have been found to be associated with a decreased risk of ovarian cancer. When we included these as additional adjustment variables in the models, we found a significant attenuation of the association between health care use and the risk of ovarian cancer, which suggests that this association may be in part mediated by these variables. This effect was also observed for different histologic subtypes. Although the inclusion of these potential causal intermediates resulted in a substantial reduction in the risk of ovarian cancer, there was still a significant increase in risk, which suggests that the risk of ovarian cancer associated with a lack of health care use is likely multifactorial and not solely dependent on the modification of risk factors.

Several epidemiologic studies have shown that women with benign ovarian neoplasms are at increased risk of developing ovarian cancer. ${ }^{30-32}$ In addition, a study of pathologic specimens has suggested that certain benign ovarian tumours may have the potential for malignant transformation. ${ }^{33}$ In light of these studies, the association between not having a pelvic examination and increased risk of ovarian cancer may be explained in part by the fact that benign neoplasms, if not identified and removed, could become malignant. Although this may be one potential mechanism to explain the association between medical encounter and ovarian cancer risk, this association is likely to be multifactorial and to involve unmeasurable and unknown variables.

Our findings suggest that women who do not have medical visits or pelvic examinations and who have no regular health care provider are at increased risk of ovarian cancer. Although the exact mechanism of this association is unknown, women, especially those who are postmenopausal, should be encouraged to maintain regular medical care.

This article has been peer reviewed.

From the School of Public Health (Abenhaim, Cramer), Harvard University, Boston, Mass.; the Norris Cotton Cancer Center (Titus-Ernstoff), Dartmouth-Hitchcock Medical Center, Lebanon, NH; and the Obstetrics and Gynecology Epidemiology Center (Cramer), Brigham and Women's Hospital, Harvard Medical School, Boston, Mass.

Competing interests: None declared.

Contributors: All 3 authors contributed to the study conception and design, the drafting and revising of the article and gave final approval of the manuscript submitted for publication.

Acknowledgement: This study was supported by the National Cancer Institute (grant number CA544I9).

\section{REFERENCES}

I. American Cancer Society. Cancer facts and figures 2007. Atlanta: American Cancer Society; 2007. Available: www.cancer.org/docroot/STT/content/STT_IX_Cancer_ Facts_Figures_2007.asp (accessed 2007 Feb 26).

2. Jemal A, Murray T, Ward E, et al. Cancer statistics, 2005. CA Cancer J Clin 2005;55: I0-30.

3. Szucs TD, Wyss P, Dedes KJ. Cost-effectiveness studies in ovarian cancer. Int J Gynecol Cancer 2003; I3(Suppl 2):212-9.

4. Menon U, Jacobs I. Screening for ovarian cancer. Best Pract Res Clin Obstet Gynaecol 2002;16:469-82.

5. Sato S, Yokoyama Y, Sakamoto T, et al. Usefulness of mass screening for ovarian carcinoma using transvaginal ultrasonography. Cancer 2000;89:582-8.

6. Van Nagell JR Jr, DePriest PD, Reedy MB, et al. The efficacy of transvaginal sonographic screening in asymptomatic women at risk for ovarian cancer. Gynecol Oncol 2000;77:350-6.

7. Jacobs IJ, Skates SJ, MacDonald N, et al. Screening for ovarian cancer: a pilot randomised controlled trial. Lancet 1999;353:1207-I0.

8. Jacobs I, Davies AP, Bridges J, et al. Prevalence screening for ovarian cancer in postmenopausal women by CA 125 measurement and ultrasonography. BMJ I993; 306:1030-4.

9. American College of Obstetricians and Gynecologists. ACOG committee opinion number 280, December 2002. The role of the generalist obstetrician-gynecologist in the early detection of ovarian cancer. Obstet Gynecol 2002;100:I4I3-6.

Io. Brenner H, Hakulinen T. Period estimates of cancer patient survival are more upto-date than complete estimates even at comparable levels of precision. J Clin Epidemiol 2006;59:570-5.

II. Silcocks PB, Robinson D. Completeness of ascertainment by cancer registries: putting bounds on the number of missing cases. J Public Health (Oxf) 2004;26:I6I-7.

I2. Bohlke K, Harlow BL, Cramer DW, et al. Evaluation of a population roster as a source of population controls: the Massachusetts Resident Lists. Am J Epidemiol I999;150:354-8.

13. Lynch CF, Logsden-Sackett N, Edwards SL, et al. The driver's license list as a population-based sampling frame in Iowa. Am J Public Health $1994 ; 84: 469-72$.

14. Cramer DW, Harlow BL, Titus-Ernstoff L, et al. Over-the-counter analgesics and risk of ovarian cancer. Lancet I998;35I:I04-7.

I5. Salganicoff A, Beckerman JZ, Wyn R, et al. Women's health in the United States: Health coverage and access to care. Menlo Park (CA): Henry J. Kaiser Family Foundation; 2002. Available: www.kff.org/womenshealth/loader.cfm?url=/commonspot/security/getfile.cfm\&PageID=I4I53 (accessed 2007 Jan 3I).

I6. Marchbanks PA, Wilson H, Bastos E, et al. Cigarette smoking and epithelial ovarian cancer by histologic type. Obstet Gynecol 2000;95:255-6o.

I7. Cramer DW. Perineal talc exposure and subsequent epithelial ovarian cancer: a case-control study. Obstet Gynecol I999;94:I60-I.

I8. Gertig DM, Hunter DJ, Cramer DW, et al. Prospective study of talc use and ovarian cancer. J Natl Cancer Inst 2000;92:249-52.

19. Kuper H, Cramer DW, Titus-Ernstoff L. Risk of ovarian cancer in the United States in relation to anthropometric measures: Does the association depend on menopausal status? Cancer Causes Control 2002;13:455-63.

20. Glud E, Kjaer SK, Thomsen BL, et al. Hormone therapy and the impact of estrogen intake on the risk of ovarian cancer. Arch Intern Med 2004;164:2253-9. 
2I. Bakken K, Alsaker E, Eggen AE, et al. Hormone replacement therapy and incidence of hormone-dependent cancers in the Norwegian women and cancer study. Int J Cancer 2004; II2:130-4.

22. Green A, Purdie D, Bain C, et al. Tubal sterilisation, hysterectomy and decreased risk of ovarian cancer. Survey of Women's Health Study Group. Int J Cancer 1997; 7I:948-5I.

23. Kjaer SK, Mellemkjaer L, Brinton LA, et al. Tubal sterilization and risk of ovarian, endometrial and cervical cancer. A Danish population-based follow-up study of more than 65000 sterilized women. Int J Epidemiol 2004;33:596-6o2.

24. Siskind V, Green A, Bain C, et al. Beyond ovulation: oral contraceptives and epithelial ovarian cancer [see comment]. Epidemiology 2000;II:I06-Io.

25. Bosetti C, Negri E, Trichopoulos D, et al. Long-term effects of oral contraceptives on ovarian cancer risk. Int J Cancer 2002;102:262-5.

26. Fleischauer AT, Olson SH, Mignone L, et al. Dietary antioxidants, supplements, and risk of epithelial ovarian cancer. Nutr Cancer 2001;40:92-8.

27. Cramer DW, Kuper H, Harlow BL, et al. Carotenoids, antioxidants and ovarian cancer risk in pre- and postmenopausal women. Int J Cancer 200I;94:128-34.

28. Wong WW, Dimitroulakos J, Minden MD, et al. HMG-CoA reductase inhibitors and the malignant cell: the statin family of drugs as triggers of tumor-specific apoptosis. Leukemia 2002;16:508-19.
29. Splichal JE, Stamm JA, Ornstein DL. The statins: multifunctional antithrombotic and antineoplastic drugs. Semin Thromb Hemost 2003;29:259-74.

30. Risch HA, Marrett LD, Jain M, et al. Differences in risk factors for epithelial ovarian cancer by histologic type. Results of a case-control study. Am J Epidemiol 1996; I $44: 363-72$.

3I. Brinton LA, Gridley G, Persson I, et al. Cancer risk after a hospital discharge diagnosis of endometriosis [see comment]. Am J Obstet Gynecol I997;176:572-9.

32. Borgfeldt $\mathrm{C}$, Andolf $\mathrm{E}$. Cancer risk after hospital discharge diagnosis of benign ovarian cysts and endometriosis. Acta Obstet Gynecol Scand 2004;83:395-400.

33. Puls LE, Powell DE, DePriest PD, et al. Transition from benign to malignant epithelium in mucinous and serous ovarian cystadenocarcinoma. Gynecol Oncol I992;47:53-7.

Correspondence to: Dr. Haim A. Abenhaim, Obstetrics and Gynecology, Sainte-Justine Hospital, 3175 chemin de la Côte Sainte-Catherine, Montreal, QC $\mathrm{H}_{3} \mathrm{~T}_{1} \mathrm{C}_{5}$;

haim.abenhaim@post.harvard.edu 